\title{
QPRT Acts as an Independent Prognostic Factor in Invasive Breast Cancer
}

\author{
Lili Zhou $\mathbb{D}^{D}$, Lin Mu $\left(\mathbb{D}\right.$, Wenyan Jiang $\mathbb{D}^{\mathbb{D}}$, and Qi Yang \\ Department of Radiology, First Hospital of Jilin University, Changchun, Jilin 130031, China \\ Correspondence should be addressed to Qi Yang; yangqi_tt@jlu.edu.cn
}

Received 8 November 2021; Accepted 22 December 2021; Published 24 February 2022

Academic Editor: Guo Chen

Copyright (C) 2022 Lili Zhou et al. This is an open access article distributed under the Creative Commons Attribution License, which permits unrestricted use, distribution, and reproduction in any medium, provided the original work is properly cited.

Background. Quinolinic acid phosphoribosyltransferase (QPRT) is a rate-limiting enzyme that encodes the uronic acid pathway, which is involved in cell cycle progression and cancer cell metabolism. Some studies have demonstrated the progrowth effect of QPRT on breast cancer (BRCA) tumour cells, but its mechanism of action requires further exploration. Methods. We investigated the expression of QPRT and the prognosis of patients with different tumours by performing a pan-cancer analysis of QPRT. Prognostic values for overall survival (OS) were determined using uni- and multivariate Cox proportional hazard analyses. The prognostic survival of patients with a different pathological staging of BRCA and with QPRT high and low expression was also analysed. We also explored the relevant pathways by which QPRT affected BRCA tumorigenesis by gene set enrichment analysis (GSEA) and western blotting. The impact of QPRT on the PI3K/Akt pathway was also evaluated. Results. Pan-cancer analysis revealed significant QPRT expression in pan-cancer and correlated with prognosis in most tumour patients. QPRT was also highly expressed in BRCA when patients had poor prognoses, and its expression was associated with different pathological BRCA subtypes. GSEA revealed an association between BRCA progression and the cell cycle and the phosphatidylinositol 3-kinase (PI3K)/Akt signalling pathway, and this association was confirmed by western blotting. Conclusion. QPRT is highly expressed in breast cancer and particularly in HER2 breast cancer. Upregulated QPRT expression is an independent predictor of breast cancer prognosis and promotes breast cancer progression by activating the PI3K/Akt signalling pathway.

\section{Introduction}

As of 2020, invasive breast cancer (BRCA) (2.26 million cases) remains one of the most commonly diagnosed cancer types worldwide [1] and is the leading cause of cancer death in women aged 20-59 years [2]. Currently, standard screening methods for BRCA include mammography, magnetic resonance imaging (MRI), computed tomography (CT), and biopsy [3]. Despite significant advances in diagnostic tools and treatment strategies, BRCA continues to rise in prevalence and affects approximately one in twenty countries worldwide [4], with higher rates in developed countries [5]. BRCA is classified, based on differences in gene expression patterns, into five major categories, luminal A, luminal B, HER2 overexpression, basal_like, and normal_like, with HER2 overexpressing breast tumours having the poorest prognosis [6]. BRCA is metastatic cancer and can spread to distant organs, such as the bone, liver, and lung, a condition that is often incurable, whereas early diagnosed BRCA generally has a better prognosis and survival rate [7]. The 5-year survival rate of patients with stage I BRCA can be as high as $100 \%$, while in patients with stage IV BRCA, it decreases to $26 \%$ [8].

In BRCA cells, a reduction in cellular levels of nicotinamide adenine dinucleotide $(\mathrm{NAD}+)$ may induce apoptosis [9]. NAD+ is a critical coenzyme involved in the redox reactions of cancer cell metabolic pathways [10] and plays a role in DNA repair, gene transcription regulation, the cell cycle, apoptosis, metabolism, and other biological processes [11]. The production of NAD+ is promoted by the activity of quinolinate phosphoribosyltransferase (QPRT), the ratelimiting enzyme encoding the kynurenic pathway, via catabolism tryptophan. QPRT is upregulated in cancer cells, and this upregulation is resistant to inhibitors of nicotinamide phosphoribosyltransferase (NAMPT) [12], the ratelimiting enzyme of the NAD + salvage pathway [13]. Studies have shown that high expression of NAMPT is related to the 
aggressive biological characteristics of BRCA [14] and can regulate the PI3K-AKT signalling pathway and promote tumour cell proliferation [15]. Similarly, QPRT expression has shown great relevance to the migration and invasive ability of BRCA cancer cells [16], and tumour xenograft assays have demonstrated the growth-promoting effect of QPRT overexpression in BRCA tumour cells [17].

The mechanism of action of QPRT on BRCA onset and progression has not been adequately studied. Therefore, the present study aimed to analyse the impact of QPRT on the expression and prognosis of BRCA tumours. The biological functions and pathways of QPRT were studied through gene set enrichment analysis (GSEA), the relationship between QPRT and signalling pathways was established by western blot analysis, and the mechanism of QPRT effects on breast cancer progression was determined.

\section{Materials and Methods}

2.1. Data Sources and Processing. Data were obtained from The Cancer Genome Atlas (TCGA) dataset, Cancer Cell Line Encyclopedia (CCLE), and Genotype-Tissue Expression (GTEx) dataset, including clinical information data and gene expression matrices for normal tissues, tumour tissues, and tumour cell lines. In total, 33 tumour samples were obtained from the TCGA dataset, RNA sequencing data for 21 tumour cell lines were obtained from the CCLE dataset, and expression profile data were obtained for 27 cancer and paracancer tissues by integrating the TCGA and GTEx datasets. The expression matrices of GSE46563 and GSE59246 were obtained from the GEO database (https://www.ncbi.nlm.nih.gov/geo/). GSE46563 contains 75 HER - and 19 HER+ samples, and GSE59246 contains a total of 50 HER- and 19 HER+ samples.

2.2. Analysis of QPRT Expression and Survival in Each Tumour. The Kruskal-Wallis test was used to analyse differences in tumour tissues and normal tissues. Survival analysis was performed using the $\mathrm{R}$ survival package, and the disease-specific survival (DFS) between QPRT expression and patients with different tumours was examined using one-way Cox risk proportional regression analysis, with data expressed as forest plots. Kaplan-Meier (KM) analysis was used to test the association between QPRT and survival among patients with different tumours. A value of $P<0.05$ was considered statistically significant.

\subsection{Expression and Survival Analysis of QPRT in BRCA.} The differences in QPRT expressions in different pathological subtypes of BRCA were analysed using the R package Limma. The log-rank test was used to test the survival differences between the high and low QPRT expression groups, and KM curves were plotted to show the overall survival (OS) and progression-free survival (PFS) of different pathological staging. Univariate and multifactorial Cox risk proportional regression analyses were performed to compare the relationship between QPRT expression and each clinicopathological feature with breast cancer survival for BRCA. The R package "RMS" was also used to plot nomograms for 1,3 , and 5 year survival rates.
2.4. Immunohistochemistry (IHC) to Detect Protein Expression. Immunohistochemical staining results of QPRT protein in breast cancer tumor tissues and normal tissues were compared using human Protein Atlas (HPA) database.

2.5. Functional and Pathway Enrichment Analyses. Based on the median expression of QPRT in breast cancer for high and low expression groups, gene ontology (GO) and Kyoto Encyclopedia of Genes and Genomes (KEGG) functional and pathway enrichment analyses were performed using the $\mathrm{R}$ package clusterProfiler. GSEA was used to demonstrate the activation or repression of biological pathways mediated by QPRT expression [18] and was performed using the $\mathrm{R}$ package clusterProfiler to search for potential biological mechanisms of QPRT in breast cancer. Biological pathway enrichment of high and low QPRT expressions was analysed using the Reactome gene sets in GSEA.

2.6. Cell Culture and Transfection. Human breast cancer MDA-MB-231 cell line (ATCC ${ }^{\circledast}$ HTB-26 $6^{\text {TM }}$ ) was cultured in Dulbecco's Modified Eagle Medium (DMEM-high glucose, 01052-1A, Biological Industries, Beit HaEmek, Israel) containing 5\% FBS (04-001-1A, Biological Industries, Beit HaEmek, Israel) and $4 \mathrm{mM}$ glutamic acid base, incubated at $37^{\circ} \mathrm{C}$ in a humidified atmosphere of $5 \% \mathrm{CO}_{2}$. Lentivirus vector is constructed (objective: HBLV-h-QPRT-ZsGreen-PURO, control: HBLV-hZsGreen-PURO), plasmid extraction kit (DP117, TIANGEN BIOTECH CO., LTD, China) was used to extract the plasmids, and $293 \mathrm{~T}$ cells were co-transfected with Lipofectamine $3000 \mathrm{kit}$ (L3000001, Thermo, USA). After infection, MDA-MB-231 cells were infected with the virus supernatant. After infection, the fusion rate of cells reached $80-90 \%$, the cells were transferred to petri dishes, and $0.5 \mu \mathrm{g} / \mathrm{mL}$ puromycin was added to screen positive cells under pressure. When the fluorescence rate and survival rate of cells were better than $95 \%$, cell lines with stable expression were obtained.

2.7. Western Blotting (WB). Cells were lysed in RIPA buffer (50 mM Tris- $\mathrm{HCl}, \mathrm{pH} 7.4,150 \mathrm{mM} \mathrm{NaCl}, 1 \%$ sodium deoxycholate, $1 \%$ NP-40, $0.1 \%$ SDS, $100 \mathrm{mM}$ PMSF, $1 \mathrm{mM}$ pepstatin $\mathrm{A}$, and $1 \mathrm{mM}$ E64). The released proteins were separated on an 8-12\% SDS polyacrylamide gel, transferred to a PVDF membrane (IPFL00010, Millipore, Burlington, MA, USA), and treated with a primary antibody. The specific primary antibodies are as follows: QPRT (ab171944, rabbit monoclonal antibody, dilution 1:1000), Akt (ab8805, rabbit polyclonal antibody, dilution 1:500), P-Akt (ab38449, rabbit polyclonal antibody, dilution 1:500), PI3K (ab32089, rabbit monoclonal antibody, dilution $1: 1000$ ), P-PI3K (ab278545, rabbit monoclonal antibody, $0.5 \mu \mathrm{g} / \mathrm{ml}$ ), MDM2 (ab16895, mouse monoclonal antibody, used at an assay-dependent concentration), P-MDM2 (ab170880, rabbit monoclonal antibody, dilution 1:50000), and $\beta$-actin (ab8226, mouse monoclonal antibody, $1 \mu \mathrm{g} / \mathrm{ml}$ ). Then the primary antibody was incubated at $4^{\circ} \mathrm{C}$ overnight, and the TBST buffer (100 mM TrIS- $\mathrm{HCl}, \mathrm{pH} 7.5,150 \mathrm{mM} \mathrm{NaCl})$ was oscillated and washed for 3 times, 5 minutes each time. The second antibody was incubated at room temperature for $1 \mathrm{~h}$, and 
the film was washed 3 times with TBST oscillation for 5 minutes each time. After the membrane was incubated with TMB substrate for 1 minute, the membrane was soaked in developing solution until the strip was clear and cleaned with tap water. Then the membrane was fixed with fixing solution, and the imaging was observed with a gel imaging analysis system $(\mathrm{XR}+$, Bio-RAD Laboratories, China).

2.8. Statistical Analysis. Statistical analysis was carried out using SPSS software (version 20.0, SPSS Inc., Chicago, IL, USA). Data were expressed as mean $\pm S D$. Student's $t$-test was employed to determine $p$ values. The $\chi^{2}$ test and Fisher's exact test were employed to assess the association between factors. Survival curves were created by the Kaplan-Meier method and compared by the log-rank tests. Multivariate survival analysis was conducted with the multivariate Cox proportional hazard regression model. Significant difference was recognized at $P<0.05$.

\section{Results}

3.1. QPRT Was Significantly Expressed in Most Tumour Tissues. Analysis of the CCLE dataset showed that QPRT was significantly expressed in all 21 tumour cell lines (Figure 1(a)). Integration of data in TCGA and GTEx revealed upregulation of QPRT expression in 16 tumours, including BRCA, COAD, and GBM, and downregulation in 10 tumours, including $\mathrm{CHOL}, \mathrm{KICH}$, and KIRP, among 27 tumour types.

\subsection{QPRT Was Associated with the Prognosis of Certain} Tumours. High QPRT expression was significantly associated with poor OS prognosis in patients with BRCA, KIRP, LGG, SKCM, and UVM, and the relationship between high and low QPRT expressions and patients with each tumour was further confirmed using KM curves (Figures 2(a) and 2(b)). To avoid the impact of nontumour death during follow-up, the relationship between QPRT expression levels and prognostic DSS (disease-specific survival) was analysed, and QPRT was found to be prognostically significant only with BRCA, KIRP, LGG, and READ tumours (Figure 2(c)). QPRT was hypothesized to be a prognostic marker for tumour DSS based on the KM curve (Figure 2(d)).

\subsection{QPRT Expression Was Significantly Associated with} Different Pathological Staging of BRCA. QPRT expression was significantly correlated with poor prognosis in BRCA patients, and QPRT expression was significantly higher in BRCA tumour tissues than in normal tissues (Figure 3(a)). Subsequent analysis of expression in different pathological subtypes of BRCA revealed significant differences in QPRT expression in all pathological subtypes, with the highest expression in the HER2 type (Figure 3(b)). The data analysis in the two validation sets (GSE46563 and GSE59246) revealed a significant differential expression of QPRT in HER2 breast cancer (Figures 3(c) and $3(\mathrm{~d})$ ). The immunohistochemical results (Figure 3(e)) showed that QPRT was localized in the cytoplasm, cell membrane, and nucleus and showed a positive expression in pathological breast cancer tissues but not in normal tissues.
3.4. The Prognosis of BRCA Was Significantly Associated with Many Factors. Survival analysis showed that breast cancer patients with low QPRT expression had higher OS and PFS than those with high expression (Figures 4(a) and 4(b)). Survival analysis of different pathological staging of BRCA also showed that the median survival time was significantly longer in basal-like and HER2-enriched than in luminal A, luminal B, and normal-like (Figure 4(c)). In addition, high QPRT expression was significantly associated with the prognosis of breast cancer patients with different pTNM stages (Figure 4(e)).

We also developed a prognostic model for BRCA to assess the impact of each factor on survival. In univariate survival analyses, BRCA cases with high QPRT expression had a poor OS. In Cox risk proportional regression analysis, after adapting for age, grade, tumour size, and subtype, QPRT was still an independent prognostic factor for OS (Figures 5(a) and 5(b)). Columnar tables were established to predict the prognostic survival of breast cancer patients at 1 , 3 , and 5 years, and the ROC curves showed that the 1-year (AUC $=0.695,95 \%$ CI: 0.599-0.791) survival prediction model was the best model (Figures 5(c) and 5(d)).

3.5. QPRT May Be Involved in BRCA Progression through the PI3K/Akt Signalling Pathway. Enrichment analysis revealed (Figures 6(a) and 6(b)) that oxygenation levels and the development of the reproductive system were the most significant biological functions; the PI3K-Akt signalling pathway was the most significant KEGG pathway; some of the related functions and pathways are listed in Table 1. Subsequent GSEA showed that the oestrogen signalling pathway and cell cycle were the most significant KEGG pathways and that the cell cycle and mitosis were the most significantly related biological processes in the Reactome gene set (Figures 6(c) and 6(d)).

3.6. QPRT Activated the PI3K/Akt Signalling Pathway in Breast Cancer Cells. We confirmed the influence of QPRT on the PI3K/Akt signalling pathway by western blotting (Figure 7). Phosphorylation and protein levels of P-PI3K (ab278545) and P-Akt (ab38449) were significantly increased in MDA-MB-231 cells with foreign expression of QPRT. We also evaluated the PI3K/Akt downstream protein kinase P-MDM2 (ab170880) and found a significant enhancement of its phosphorylation and protein levels. These results indicate that QPRT may promote breast cancer progression through the PI3K/Akt pathway.

\section{Discussion}

Cancer is a significant factor affecting the health and longevity of people worldwide, with nearly 20 million new cancer cases and nearly 10 million deaths reported worldwide in 2020 [19]. Pan-cancer analysis can identify the similarities and differences in the tumour genomes and provide helpful information for cancer diagnosis and treatment [20]. In the present study, we evaluated the efficacy of QPRT for pan-cancer analysis. QPRT is significantly expressed in most tumours, and its expression is related to prognosis. Among them, the expression of QPRT has a prominent effect on the prognosis of breast cancer patients. 


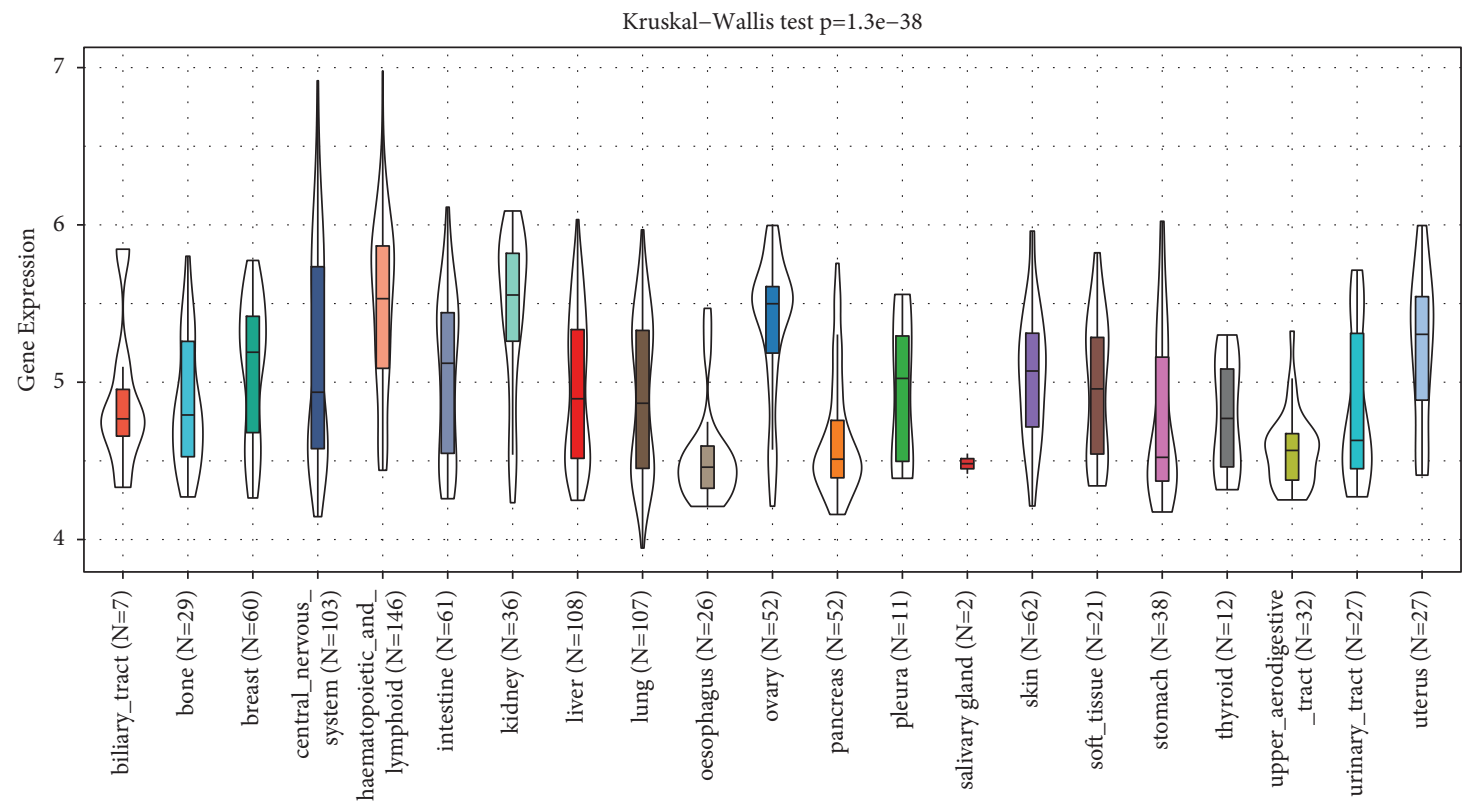

(a)

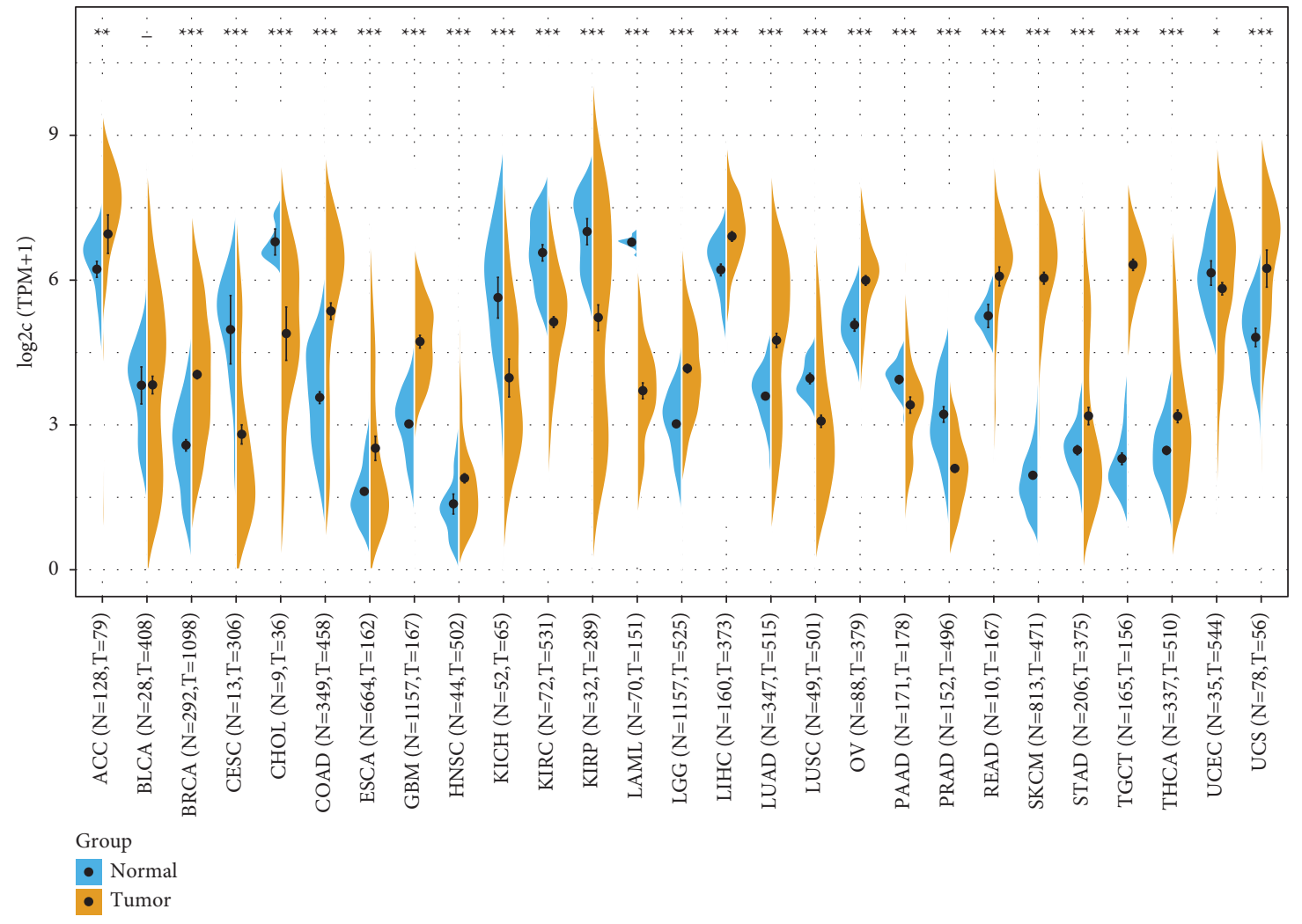

(b)

FIgURE 1: Expression levels of QPRT in different tumours: (a) QPRT expression in the CCLE dataset, total 21 tumour cell lines; (b) QPRT expression in the integrated GTEx and TCGA dataset, total 27 tumors. ${ }^{*} P<0.01,{ }^{* *} P<0.001$, and ${ }^{* * *} P<0.0001$.

Because of the strong relationship found for QPRT in breast cancer, this study mainly analysed the relationship between QPRT and breast cancer. The TCGA dataset as the test set revealed significant QPRT expression in breast cancer, especially HER2 breast cancer. These results were reproduced in two GEO validation sets. A study screening for prognosis-related candidate genes in breast cancer showed that QPRT expression was significantly associated with prognosis in breast cancer patients [21]. The analysis in the present study shows that QPRT is an independent prognostic factor of breast cancer and is related to different pathological subtypes. 


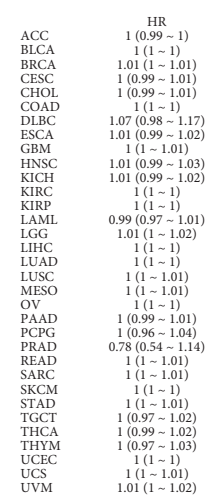

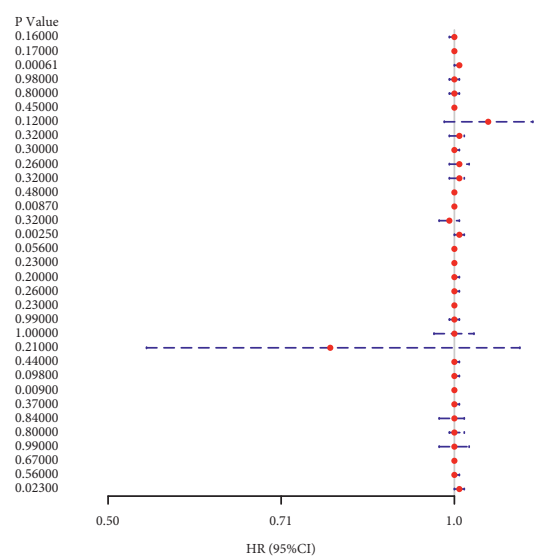

(a)

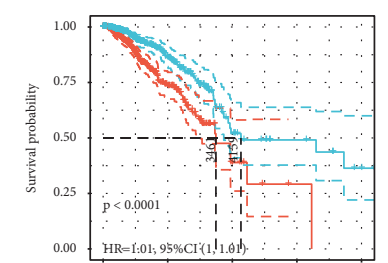

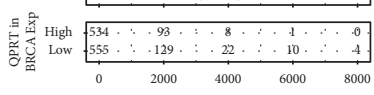
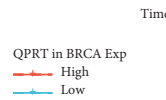

(c)

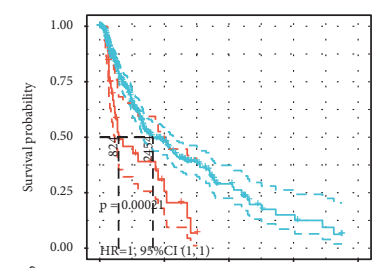

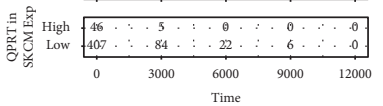

QPRT in SKCM Exp

\pm High
$\simeq$ Low

(f)

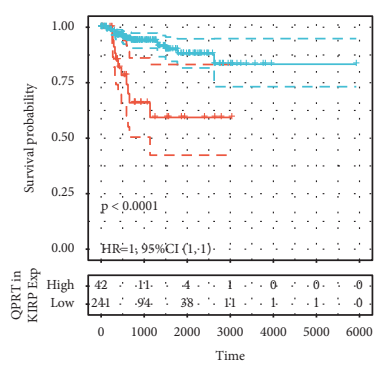

QPRT in KIRP Exp

$\longleftarrow$ High
$\simeq$ Low

(i)

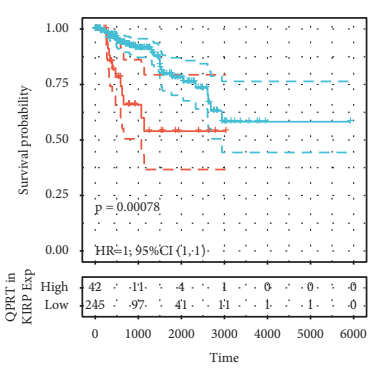

QPRT in KIRP Exp

(d)

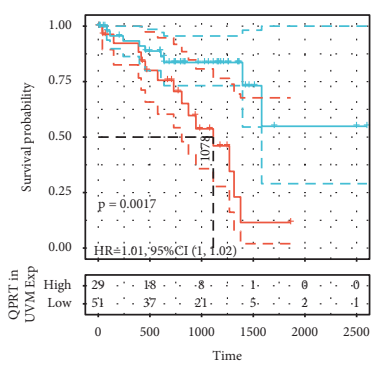

QPRT in UVM Exp

$\longrightarrow$ High

(g)

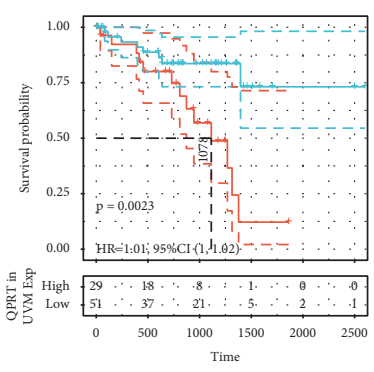

QPRT in UVM Exp

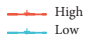

(j)

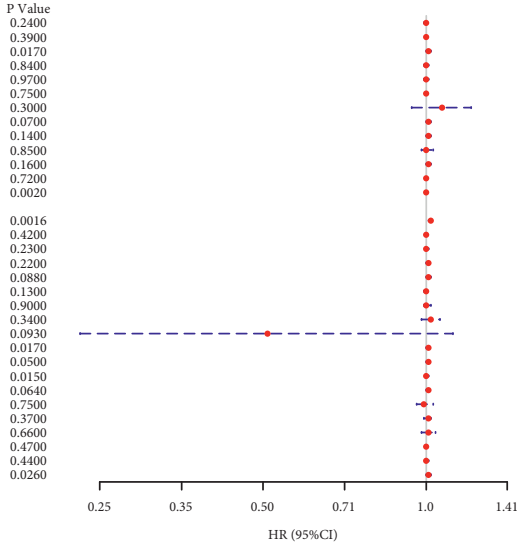

(b)

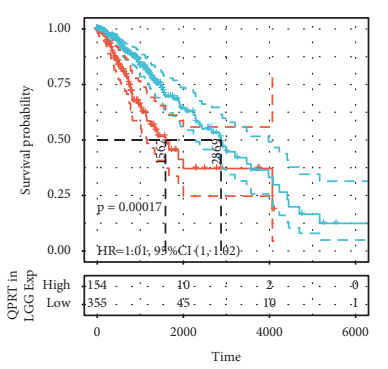

QPRT in LGG Exp
- High

(e)

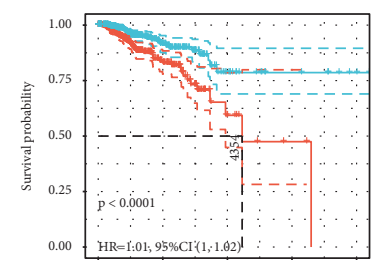

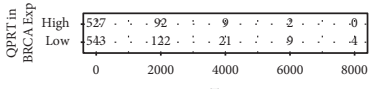

QPRT in BRCA Exp

+ High

(h)

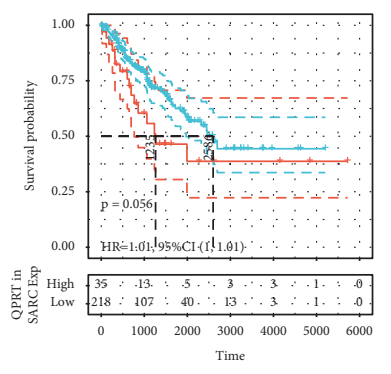

QPRT in SARC Exp

(k)

Figure 2: Continued. 


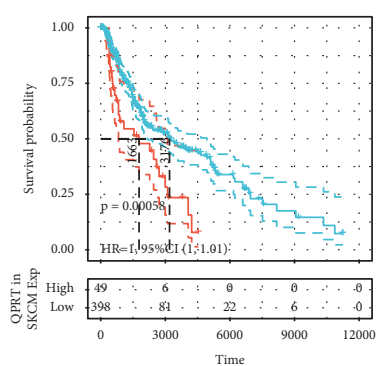

QPRT in SKCM Exp $\underset{\text { High }}{\longleftarrow \text { Low }}$

(l)

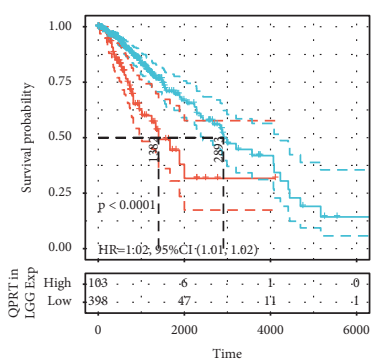

QPRT in LGG Exp

- High

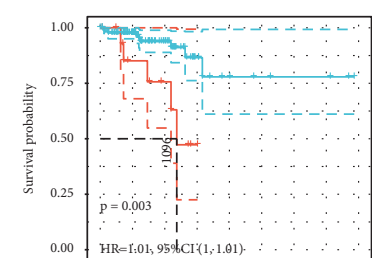

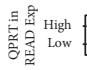

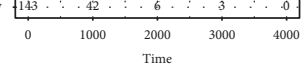

QPRT in READ Exp + High
+ Low

(n)

FIGURE 2: OS and DSS of high and low expressions of QPRT in each tumour: (a) forest plot of OS of QPRT in each tumour; (b) forest plot of DSS of QPRT in each tumour; (c-g) KM survival curve to show the OS of patients with high and low QPRT expressions in different tumors; $(\mathrm{h}-\mathrm{n})$ KM survival curve to show the DSS of patients with high and low QPRT expressions in different tumors.

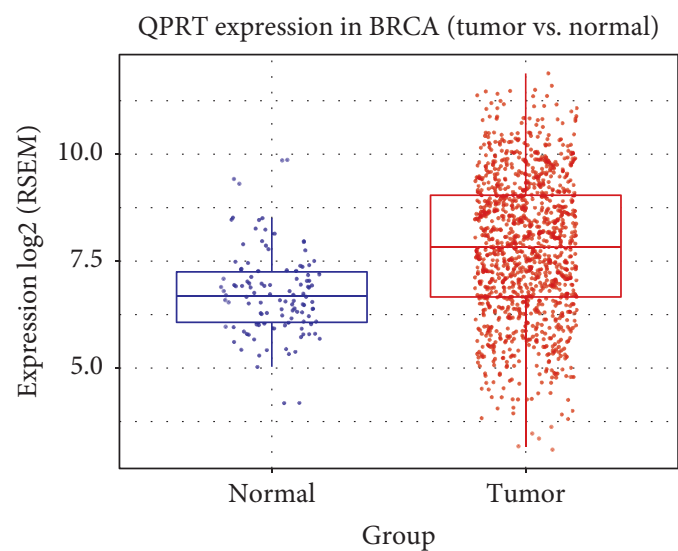

Group

Normal

Tumor

(a)

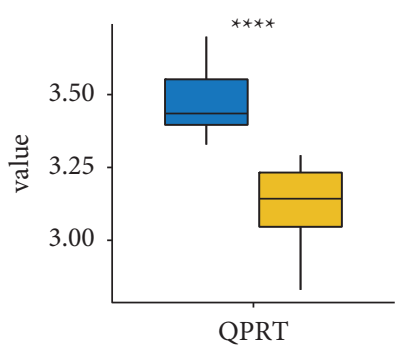

category

巨High

$\rightleftarrows$ Low

(c)

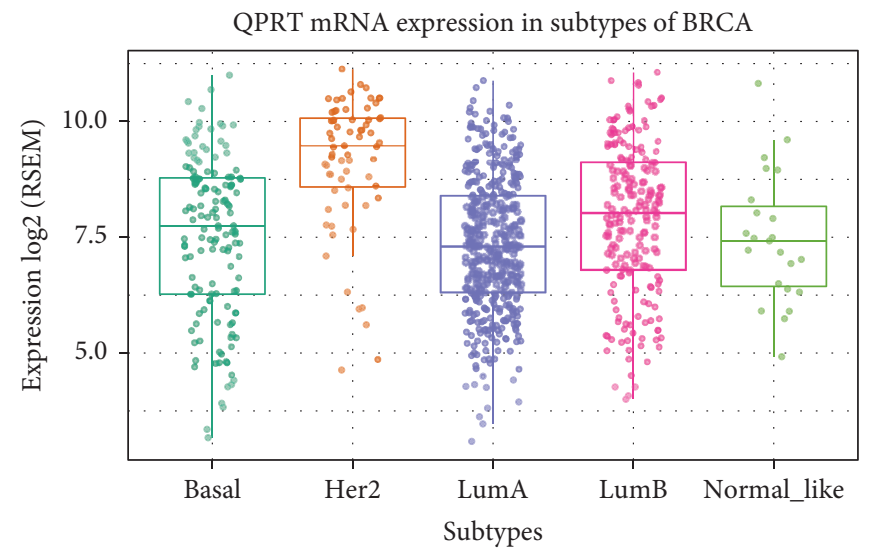

Subtypes

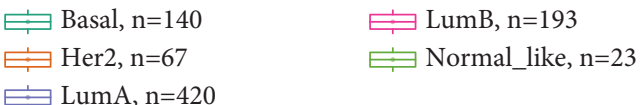

(b)

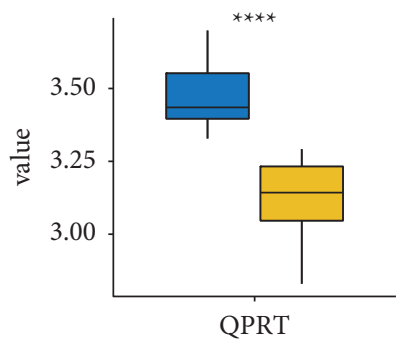

category

Е High

Low

(d)

Figure 3: Continued. 


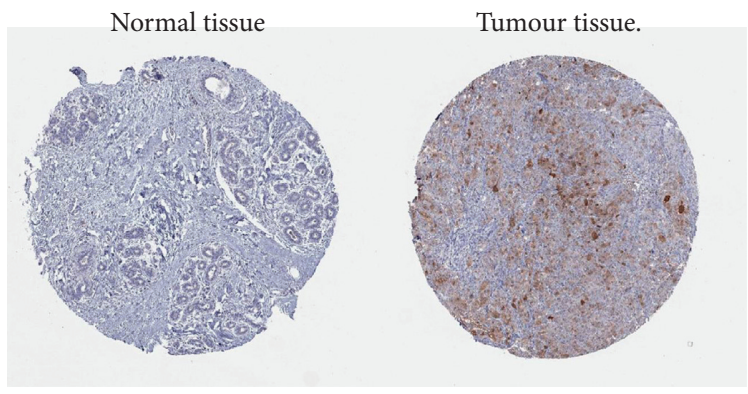

(e)

FIGURE 3: The expression of QPRT in BRCA: (a) differential expression of QPRT in BRCA tumour tissues and normal tissues; (b) expression of QPRT in different pathological staging of BRCA; ((c), (d)) the high and low expression distribution of QPRT in HER2 breast cancer, the data come from GSE46563 and GSE59246, respectively; (e) breast cancer immunohistochemical map.

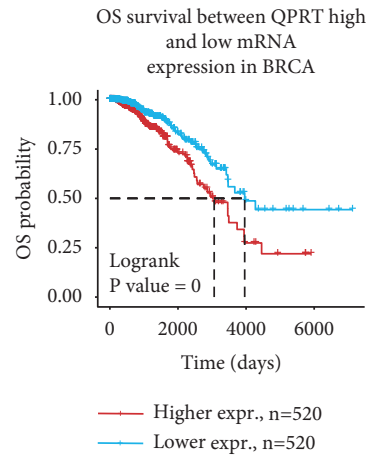

(a)
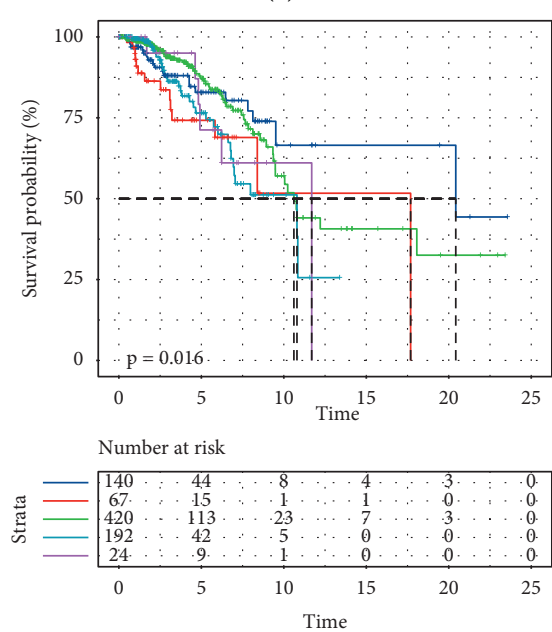

Strata

$$
\begin{aligned}
& \square \text { Group=Basal-like } \\
& \square \text { Group=HER2-enriched } \\
& \square \text { Group=Luminal A } \\
& \square \text { Group=Luminal B } \\
& \square \text { Group=Normal-like }
\end{aligned}
$$

(c)

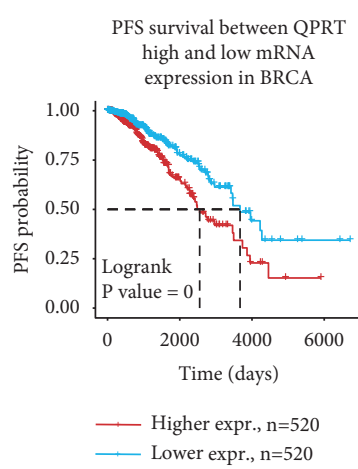

(b)
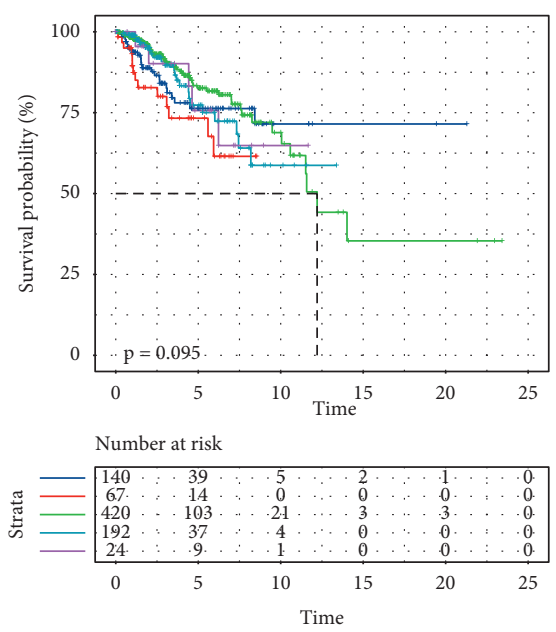

Strata

$$
\begin{aligned}
& \longleftarrow \text { Group=Basal-like } \\
& \square \text { Group=HER2-enriched } \\
& \square \text { Group=Luminal A } \\
& \square \text { Group=Luminal B } \\
& \square \text { Group=Normal-like }
\end{aligned}
$$

(d)

Figure 4: Continued. 


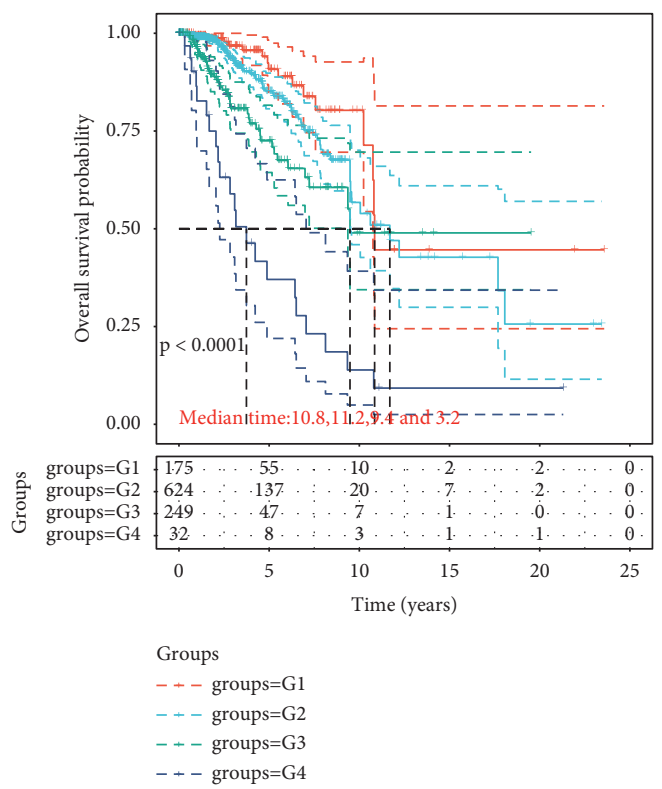

(e)

Figure 4: Prognostic relationship between QPRT and BRCA: (a) the relationship between high and low QPRT expressions and OS of BRCA by KM survival analysis; (b) the relationship between high and low QPRT expressions and PFS of BRCA by KM survival analysis; (c) the relationship between QPRT and OS of different pathological staging of BRCA; (d) the relationship between QPRT and PFS of different pathological staging of BRCA; (e) the relationship between QPRT and OS of different pTNM staging of BRCA.

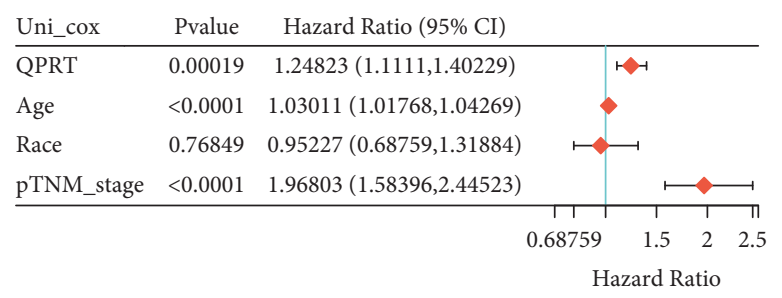

(a)

Points

QPRT

Total Points

Linear Predictor

$$
\begin{array}{lllllllll}
-0.8 & -0.6 & -0.4 & -0.2 & 0 & 0.2 & 0.4 & 0.6 & 0.8
\end{array}
$$

1-year survival Pro

3-year survival Pro

0.95

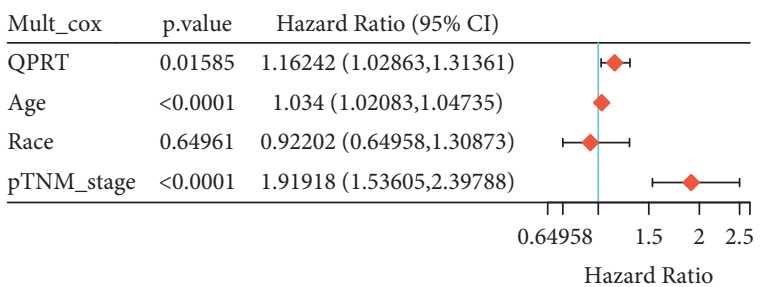

(b)
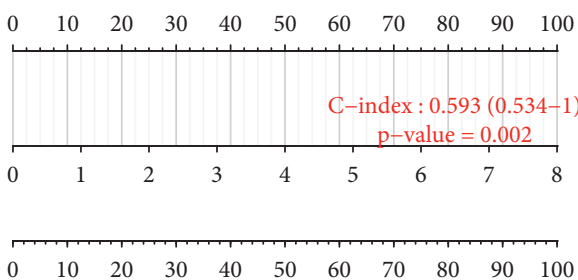

5-year survival Pro

(c)

FIgURe 5: Continued. 


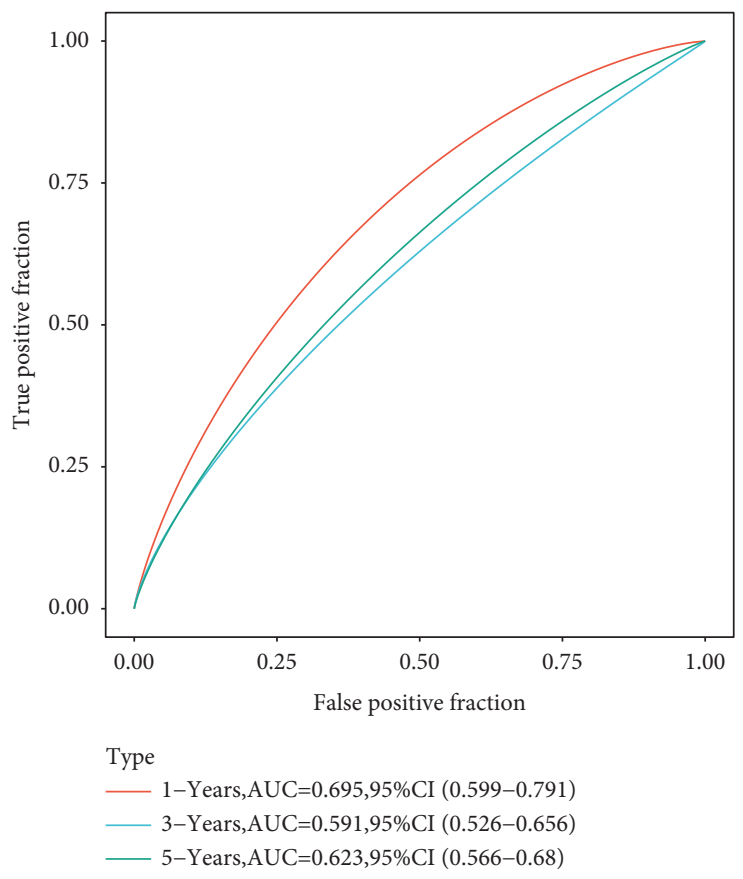

(d)

FIGURE 5: Prognosis of the BRCA prediction column line graph: (a) single-factor Cox regression analysis of the risk relationship of BRCA with age, race, and pTNM; (b) multifactor Cox regression analysis of the risk relationship of BRCA with age, race, and pTNM; (c) column line graphs for predicting the overall survival of BRCA patients at 1, 3, and 5 years; (d) ROC curve graphs for overall survival at 1,3 , and 5 years.

In breast cancer, HER2 gene amplification can lead to the proliferation of specific aggressive breast cells, and HER2 expression has been identified as an independent factor for the poor prognosis of breast cancer patients [22]. Targeted therapy is one of the treatments aimed at improving the survival rate of HER2-positive breast cancer patients, but the selection of targeted genes still needs further study [23].

QPRT catalyses the production of nicotinic acid mononucleotide (NMN), which in turn promotes the synthesis of nicotinamide adenine dinucleotide (NAD+), which plays a crucial role in cell survival [24]. Zhang et al. used in vivo and in vitro experiments to confirm that QPRT promotes growth, migration, and invasion of breast cancer and inhibits cell apoptosis [17]. Liu et al. also provided strong evidence that upregulation of QPRT promotes breast cancer progression [16]. Earlier work indicated that QPRT might have an antiapoptotic function (Ullmark et al., 2017). Furthermore, QPRT was identified as a potential prognosis biomarker of BC [21]. However, whether QPRT is an independent prognostic factor in invasive breast cancer and the mechanisms by which QPRT may contribute to invasive breast cancer remain undefined. Thus, the present study was based on this previous research and aimed to explore the mechanism underlying promoting breast cancer progression by QPRT.

QPRT overexpression is known to activate the PI3K/Akt signalling pathway in cancer cells [25], but this has not been proven in breast cancer. The GSEA results presented here showed that QPRT expression was related to the PI3K/Akt signalling pathway, and western blot analysis showed that overexpression of QPRT can increase the phosphorylation levels of PI3K and Akt, indicating that QPRT and the PI3K/ Akt signalling pathway may have a positive feedback effect in breast cancer.

Phosphoinositide 3-kinase (PI3K) can integrate signals from growth factors, cytokines, and other extracellular stimuli, and the modification of this pathway is closely related to the pathogenesis of cancer [26, 27]. Protein kinase B (PKB, also known as Akt) is an essential mediator of the PI3K pathway and the signalling endpoint of various growth factors and cytokines [28]. The PI3K/ Akt signalling pathway is one of the phosphatidylinositol signalling systems involved in tumorigenesis, cell growth, proliferation, metabolism, survival, and apoptosis [29]. The PI3K/Akt signalling pathway is activated in various cancers and has been proven to be one of the most important signalling pathways in cancer development [30]. The PI3K/Akt signalling pathway has attracted increasing attention in breast cancer research as activating this pathway can promote breast cancer cell proliferation, inhibit apoptosis [31], and modulate cell invasion [16]. Human epidermal growth factor receptor-2 (HER2) is involved in the development of breast cancer through the $\mathrm{PI} 3 \mathrm{~K} / \mathrm{Akt} / \mathrm{mTOR}$ pathway [27], and the PI3K/Akt/mTOR pathway is an important pathway involved in chemoresistance and survival of triple-negative breast cancer (TNBC) [32]. However, the current study also had some limitations. This research was based on microarray data 


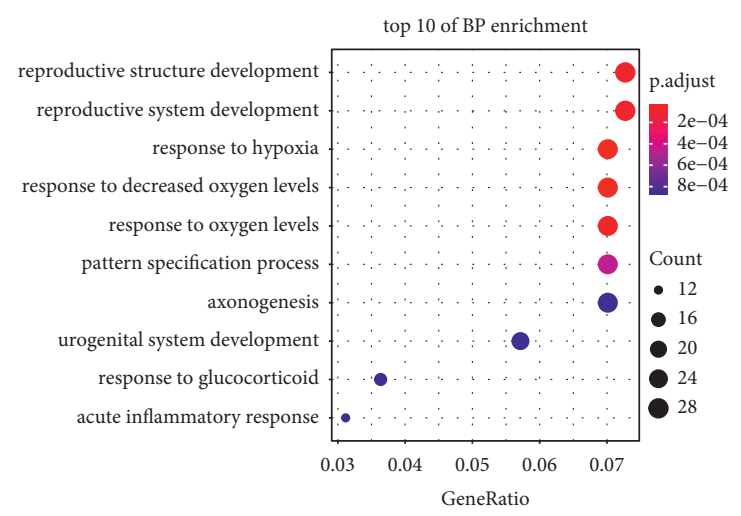

(a)

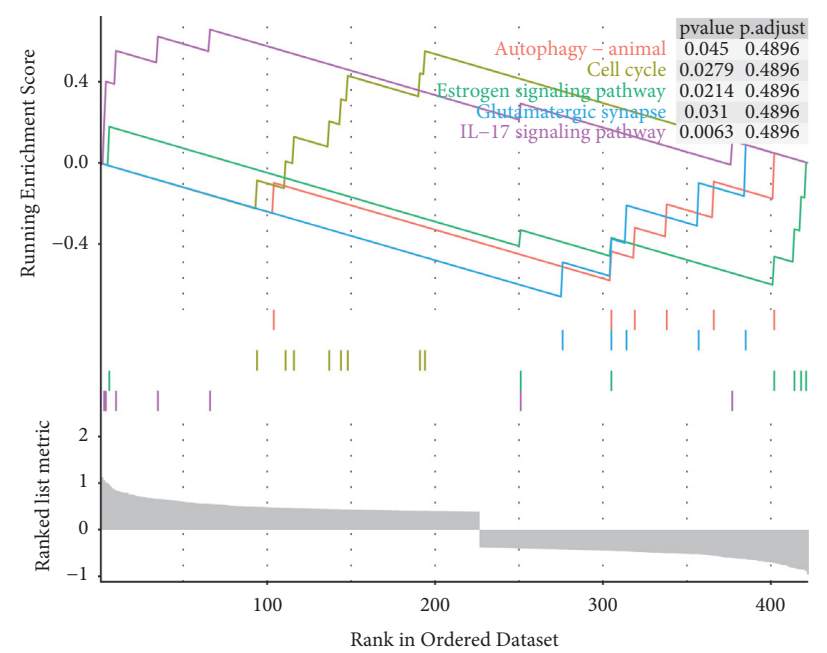

(c)

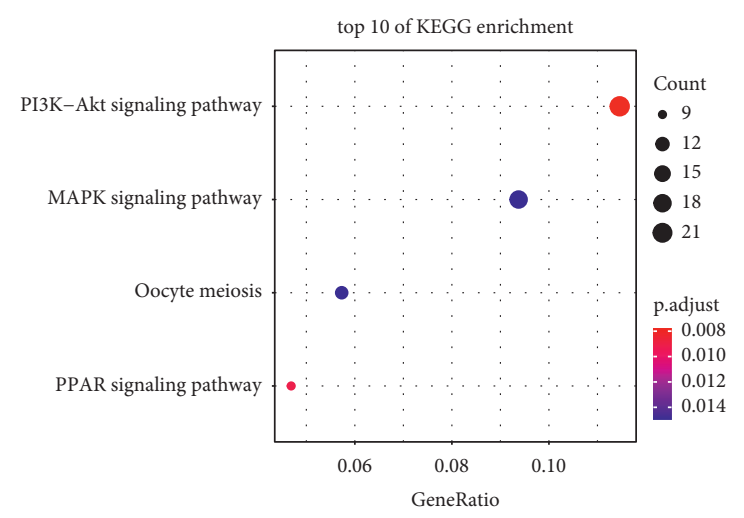

(b)

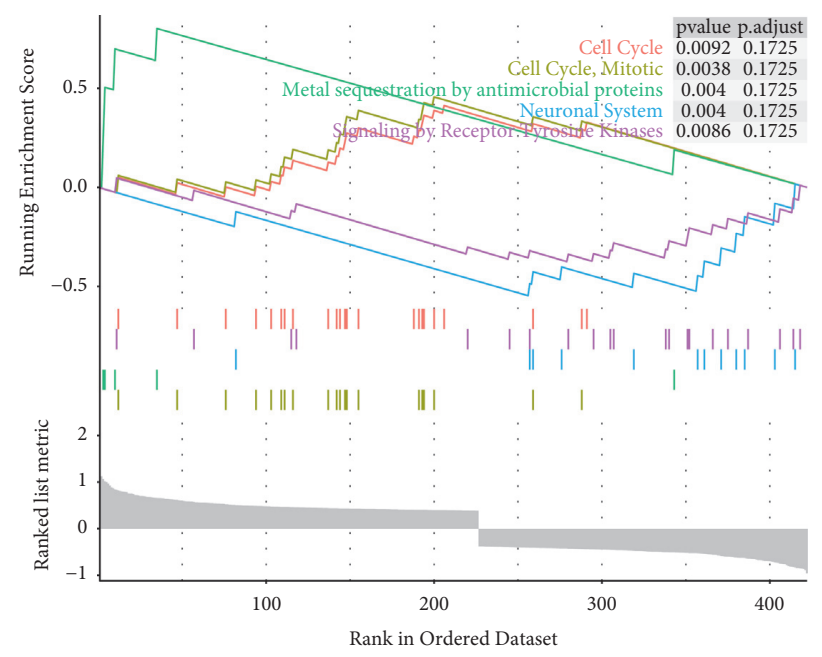

(d)

FIGURE 6: BRCA whole gene enrichment analysis: (a) enrichment of GO function in QPRT expression; (b) enrichment of KEGG function in QPRT expression; (c) enrichment of the KEGG pathway in GSEA with high and low QPRT expressions; (d) enrichment of biological processes in the Reactome gene set with high and low QPRT expressions. $P<0.05$ is statistically significant.

TABLE 1: The most significant GO biofunction and KEGG pathways in the enrichment analysis.

\begin{tabular}{lcccc}
\hline Class & Function/pathway & $p$ value & $p$. adjust & $q$-value \\
\hline & Response to hypoxia & $7.92 \mathrm{E}-09$ & $3.08 \mathrm{E}-05$ & $2.57 \mathrm{E}-05$ \\
& Response to decreased oxygen levels & $1.50 \mathrm{E}-08$ & $3.08 \mathrm{E}-05$ & $2.57 \mathrm{E}-05$ \\
& Response to oxygen levels & $5.54 \mathrm{E}-08$ & $7.58 \mathrm{E}-05$ & $6.32 \mathrm{E}-05$ \\
& Reproductive structure development & $9.59 \mathrm{E}-08$ & $9.10 \mathrm{E}-05$ & $7.58 \mathrm{E}-05$ \\
BP & Reproductive system development & $1.11 \mathrm{E}-07$ & $9.10 \mathrm{E}-05$ & 0.000450942 \\
& Pattern specification process & $6.59 \mathrm{E}-07$ & 0.000852121 & 0.00037565 \\
& Urogenital system development & $1.52 \mathrm{E}-06$ & 0.000852121 & 0.000709846 \\
& Axonogenesis & $1.66 \mathrm{E}-06$ & 0.000874411 & 0.000709846 \\
& Response to glucocorticoid & $2.04 \mathrm{E}-06$ & 0.000874411 & 0.000728415 \\
KEGG & Acute inflammatory response & $2.34 \mathrm{E}-06$ & 0.007825176 & 0.007132445 \\
& PI3K-Akt signalling pathway & $3.00 \mathrm{E}-05$ & 0.009139327 & 0.00833026 \\
& PPAR signalling pathway & $7.00 \mathrm{E}-05$ & 0.014943102 & 0.01362025 \\
& MAPK signalling pathway & 0.000198423 & 0.014943102 & 0.000229013 \\
\hline
\end{tabular}

analysis. The samples from the datasets were insufficient and without cancer stage information, and data from biological samples carried out no confirmation. Consequently, large-scale, potential, and widespread clinical examinations are required to confirm our results. It was necessary to obtain a single gene to profile QPRT expression in BRCA. The mechanism of QPRT needs further findings through in vivo and in vitro models. 


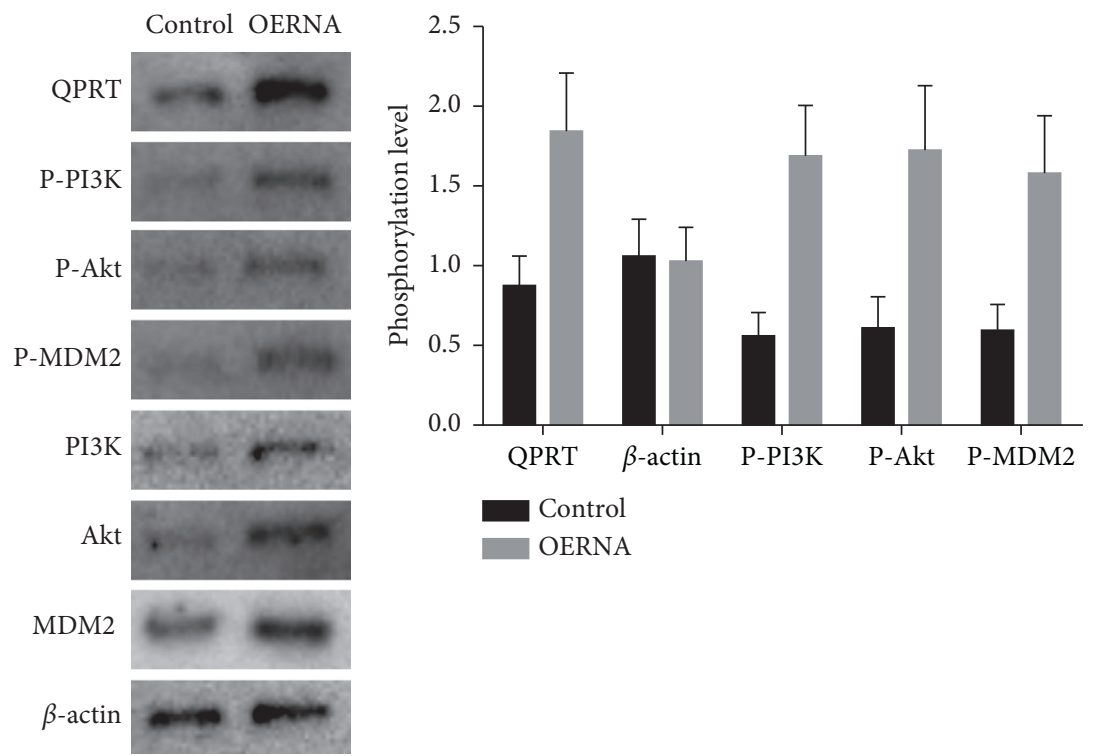

(a)

(b)

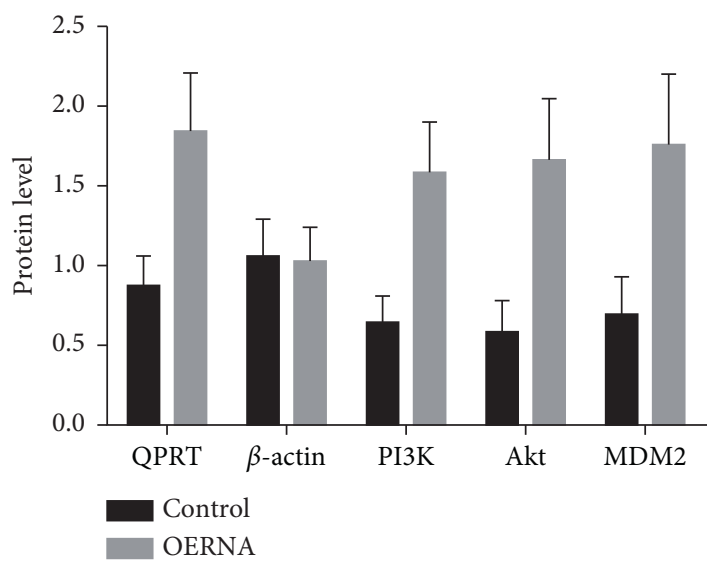

(c)

FIGURE 7: QPRT overexpression induces phosphorylation of PI3K/Akt-related signalling pathways: (a) western blot; (b) phosphorylation levels of different signalling pathways; (c) protein levels of different signalling pathways.

\section{Conclusions}

Collectively, our results here support a vital role for QPRT in breast cancer and indicate that its upregulation is related to the poor prognosis of patients with BRCA. Subsequently, in vitro experimental results show that QPRT upregulation may affect breast cancer progression by activating the PI3K/ Akt signalling pathway. The current study implies that QPRT may therefore be a novel specific therapeutic target for breast cancer treatment.

\section{Data Availability}

The data used to support the findings of this study are available from the corresponding author upon request.

\section{Conflicts of Interest}

The authors declare that they have no conflicts of interest.

\section{Acknowledgments}

This work was supported by the Finance Department of Jilin Province under grant no. JLSWSRCZX2020-069, Jilin Provincial Key Laboratory of Medical Imaging and Big Data under grant no. 20200601003JC, and Radiology and Technology Innovation Center of Jilin Province under grant no. 20190902016TC.

\section{References}

[1] J. Ferlay, M. Colombet, I. Soerjomataram et al., "Cancer statistics for the year 2020: an overview," International Journal of Cancer, vol. 149, 2021.

[2] R. L. Siegel, K. D. Miller, and A. Jemal, "Cancer statistics, 2020," CA: A Cancer Journal for Clinicians, vol. 70, no. 1, pp. 7-30, 2020.

[3] L. Wang, "Early diagnosis of breast cancer," Sensors, vol. 17, no. 7, Article ID 1572, 2017. 
[4] K. L. Britt, J. Cuzick, and K.-A. Phillips, "Key steps for effective breast cancer prevention," Nature Reviews Cancer, vol. 20, no. 8, pp. 417-436, 2020.

[5] Z. Momenimovahed and H. Salehiniya, "Epidemiological characteristics of and risk factors for breast cancer in the world," Breast Cancer: Targets and Therapy, vol. 11, pp. 151-164, 2019.

[6] X. Dai, T. Li, Z. Bai et al., "Breast cancer intrinsic subtype classification, clinical use and future trends," American journal of cancer research, vol. 5, no. 10, pp. 2929-2943, 2015.

[7] Y.-S. Sun, Z. Zhao, Z.-N. Yang et al., "Risk factors and preventions of breast cancer," International Journal of Biological Sciences, vol. 13, no. 11, pp. 1387-1397, 2017.

[8] K. D. Miller, L. Nogueira, A. B. Mariotto et al., "Cancer treatment and survivorship statistics, 2019," CA: A Cancer Journal for Clinicians, vol. 69, no. 5, pp. 363-385, 2019.

[9] Z. B. Pour, M. Nourbakhsh, K. Mousavizadeh, Z. Madjd, S. S. Ghorbanhosseini, and Z. Abdolvahabi, "Up-regulation of miR-381 inhibits NAD+ salvage pathway and promotes apoptosis in breast cancer cells," EXCLI journal, vol. 18, Article ID 683, 2019.

[10] M. Vora, J. Ansari, R. M. Shanti et al., "Increased nicotinamide phosphoribosyltransferase in rhabdomyosarcomas and leiomyosarcomas compared to skeletal and smooth muscle tissue," Anticancer Research, vol. 36, no. 2, pp. 503-507, 2016.

[11] R. Xu, Z. Yuan, L. Yang, L. Li, D. Li, and C. Lv, "Inhibition of NAMPT decreases cell growth and enhances susceptibility to oxidative stress," Oncology Reports, vol. 38, no. 3, pp. 1767-1773, 2017.

[12] J. Guo, L. T. Lam, K. L. Longenecker et al., "Identification of novel resistance mechanisms to NAMPT inhibition via the de novo NAD+ biosynthesis pathway and NAMPT mutation," Biochemical and Biophysical Research Communications, vol. 491, no. 3, pp. 681-686, 2017.

[13] A. A. Pramono, G. M. Rather, H. Herman, K. Lestari, and J. R. Bertino, "NAD- and NADPH-contributing enzymes as therapeutic targets in cancer: an overview," Biomolecules, vol. 10, no. 3, Article ID 358, 2020.

[14] Y.-C. Lee, Y.-H. Yang, J.-H. Su, H.-L. Chang, M.-F. Hou, and S.-S. F. Yuan, "High visfatin expression in breast cancer tissue is associated with poor survival," Cancer Epidemiology Biomarkers \& Prevention, vol. 20, no. 9, pp. 1892-1901, 2011.

[15] C. Ji, R. Cong, Y. Wang et al., "Relationship between NAMPT/ $\mathrm{PBEF} /$ visfatin and prognosis of patients with malignant tumors: a systematic review and meta-analysis," Annals of Translational Medicine, vol. 7, no. 23, Article ID 785, 2019.

[16] C.-L. Liu, S.-P. Cheng, M.-J. Chen, C.-H. Lin, S.-N. Chen, and Y.-H. Kuo, "Quinolinate phosphoribosyltransferase promotes invasiveness of breast cancer through myosin light chain phosphorylation," Frontiers in Endocrinology, vol. 11, Article ID 1124, 2021.

[17] Z. Yue, J. Shusheng, S. Hongtao et al., "Silencing DSCAM-AS1 suppresses the growth and invasion of ER-positive breast cancer cells by downregulating both DCTPP1 and QPRT," Aging, vol. 12, no. 14, pp. 14754-14774, 2020.

[18] Y. Zhang, D. J. Topham, J. Thakar, and X. Qiu, "FUNNELGSEA: FUNctioNal ELastic-net regression in time-course gene set enrichment analysis," Bioinformatics, vol. 33, no. 13, pp. 1944-1952, 2017.

[19] H. Sung, J. Ferlay, R. L. Siegel et al., "Global cancer statistics 2020: GLOBOCAN estimates of incidence and mortality worldwide for 36 cancers in 185 countries," CA: A Cancer Journal for Clinicians, vol. 71, no. 3, pp. 209-249, 2021.

[20] D. He, X. Zhang, and J. Tu, "Diagnostic significance and carcinogenic mechanism of pan-cancer gene POU5F1 in liver hepatocellular carcinoma," Cancer medicine, vol. 9, no. 23, pp. 8782-8800, 2020.

[21] Y.-H. Xu, J.-L. Deng, L.-P. Wang et al., "Identification of candidate genes associated with breast cancer prognosis," DNA and Cell Biology, vol. 39, no. 7, pp. 1205-1227, 2020.

[22] J. Wang and B. Xu, "Targeted therapeutic options and future perspectives for HER2-positive breast cancer," Signal transduction and targeted therapy, vol. 4, no. 1, pp. 34-22, 2019.

[23] K. Goutsouliak, J. Veeraraghavan, V. Sethunath et al., "Towards personalized treatment for early stage HER2-positive breast cancer," Nature Reviews Clinical Oncology, vol. 17, no. 4, pp. 233-250, 2020.

[24] H. S. Youn, T. G. Kim, M. K. Kim et al., "Structural insights into the quaternary catalytic mechanism of hexameric human quinolinate phosphoribosyltransferase, a key enzyme in de novo NAD biosynthesis," Scientific Reports, vol. 6, no. 1, Article ID 19681, 2016.

[25] Y. C. Niu, J. Tong, X. F. Shi, and T. Zhang, "MicroRNA-654$3 p$ enhances cisplatin sensitivity by targeting QPRT and inhibiting the PI3K/AKT signaling pathway in ovarian cancer cells," Experimental and Therapeutic Medicine, vol. 20, no. 2, pp. 1467-1479, 2020.

[26] S. Noorolyai, N. Shajari, E. Baghbani, S. Sadreddini, and B. Baradaran, "The relation between PI3K/AKT signalling pathway and cancer," Gene, vol. 698, pp. 120-128, 2019.

[27] M. A. Ortega, O. Fraile-Martínez, Á. Asúnsolo, J. Buján, N. García-Honduvilla, and S. Coca, "Signal transduction pathways in breast cancer: the important role of PI3K/Akt/ mTOR," Journal of oncology, vol. 2020, 2020.

[28] C. De Marco, C. Laudanna, N. Rinaldo et al., "Specific gene expression signatures induced by the multiple oncogenic alterations that occur within the PTEN/PI3K/AKT pathway in lung cancer," PLoS One, vol. 12, no. 6, Article ID e0178865, 2017.

[29] H. Zhang, Y. Z. Pan, M. Cheung et al., "LAMB3 mediates apoptotic, proliferative, invasive, and metastatic behaviors in pancreatic cancer by regulating the PI3K/Akt signaling pathway," Cell Death \& Disease, vol. 10, no. 3, pp. 230-314, 2019.

[30] N. Jiang, Q. Dai, X. Su, J. Fu, X. Feng, and J. Peng, "Role of PI3K/AKT pathway in cancer: the framework of malignant behavior," Molecular Biology Reports, vol. 47, no. 6, pp. 4587-4629, 2020.

[31] Z. Gholinejad, N. kheiripour, M. Nourbakhsh et al., "Extracellular NAMPT/Visfatin induces proliferation through ERK1/2 and AKT and inhibits apoptosis in breast cancer cells," Peptides, vol. 92, pp. 9-15, 2017.

[32] M. A. Khan, V. K. Jain, M. Rizwanullah, J. Ahmad, and K. Jain, "PI3K/AKT/mTOR pathway inhibitors in triple-negative breast cancer: a review on drug discovery and future challenges," Drug Discovery Today, vol. 24, no. 11, pp. 2181-2191, 2019. 